\title{
Expanding Our Knowledge of Interstellar Neutral Hydrogen Shells
}

\author{
Tyler Laszczkowski ${ }^{1}$
}

Shauna Sallmen

University of Wisconsin - La Crosse

\begin{abstract}
The purpose of this research is to increase the number of Galactic neutral hydrogen (HI) shells available for study. By identifying and studying more HI shells at various stages of their evolution, we can start to fill the gap in our understanding of the interactions of structures in the interstellar medium. This project consisted of visually identifying new HI Shells using $21-\mathrm{cm}$ data downloaded from the GALFA-HI online database. This survey has higher angular resolution than the data used in previous searches, allowing us to discover smaller features. The results include a new list of previously undiscovered shell-like HI features along with measurements of their basic properties: location, mean angular diameter, shape, signs of expansion. Upon completing the search of $1 / 7^{\text {th }}$ of the data cubes containing complete GALFA data, 141 potential shells were discovered. Additionally, one pattern appeared several times while identifying potential shells. This pattern can be described as a "figure-eight" feature composed of two features that are likely related. The completion of this project has expanded the current database of potential HI Shells in our galaxy, which can be used to provide important data in regards to the study of shell evolution and the role of shells within the ISM.
\end{abstract}

1 I would like to acknowledge the WSGC for granting me this award and allowing me this opportunity to further my education and involvement in Astronomy research. Further acknowledgments are included at the end of the paper. 


\section{Background}

The matter which makes up the interstellar medium (ISM) is full of complex, interacting features. In terms of mass, this matter is $99 \%$ gas and $1 \%$ dust with only $2 \%$ of the atoms being elements heavier than helium. In general, the ISM has a very low density, but it is far from uniform. Through winds and supernova explosions, stars send out material that is enriched with the heavy elements made in stars during their lives and deaths. Eventually, this enriched material mixes into and is distributed throughout the turbulent ISM. This allows for the formation of new stars as well as star systems. Thus the heavy elements found within our own solar system were created and distributed by these processes.

As a result of these stellar winds and supernovae, expanding bubbles of hot gas will commonly form in the ISM. As such a bubble expands; it pushes the surrounding cool neutral hydrogen into a shell around the bubble. We can observe and study these shells using the $21 \mathrm{~cm}$ radiation from neutral hydrogen atoms using radio telescopes. Over time, the shell expansion will begin to slow as it interacts with the ISM and eventually the bubble cools as it loses energy and mixes with the surrounding ISM.

While we have a general understanding of the evolution of the ISM, much is still uncertain. Various models have attempted to identify the processes and structures that contribute to the evolution of the ISM in our Galaxy, and it is clear that shell evolution plays a key role (e.g. models of McKee \& Ostriker 1977, Shapiro \& Field 1976, Slavin \& Cox 1993). As described by the galactic fountain model, superbubbles (the result of multiple supernovae) can cause hot gas to rise out of the Galactic plane where it will then cool, and fall back onto the Galaxy's disk.

However there still exist gaps in our understanding so we lack a complete picture of the details involved; for example the role of magnetic fields and exactly how energy of supernova explosions disrupts the medium (e.g. see review by Cox 2005). By identifying and studying more HI shells at various stages of their evolution, we can start to fill this gap in our understanding of the interactions of structures in the ISM. We need to study as many shells as possible in order to understand the complete picture.

Our research group works to increase our knowledge of HI Shells by discovering new shells and measuring their properties. For suitable shells, detailed follow-up study at various wavelengths increases the number of well-observed shells that can be used to study the processes of shell evolution. Using the 21-cm neutral hydrogen maps available in the SETHI (Search for Extraterrestrial HI) database (Korpela 2002, 2004), my advisor and her research students have created a list of 71 shells at various reference velocities, none of which had been previously identified (Sallmen et al., in preparation). This search resulted in shells missed by previous searches for several reasons. (1) The SETHI database had improved angular resolution compared with other 21-cm surveys away from the Galactic plane (e.g. Leiden-Dwingeloo survey, Hartmann \& Burton 1997), so smaller shells could be found. (2) Many searches identify shells by signs of expansion, so older shells expanding more slowly are absent from these catalogs (e.g. Ehlerová \& Palouš 2005). (3) The visual identification techniques that were utilized allowed for the discovery of shells that could have been missed by an automated search (fragmented, incomplete, or small shells). 
The Galactic Arecibo L-band Feed Array HI (GALFA-HI) survey (Peek et al. 2011) is producing neutral-hydrogen maps of Galactic gas with better angular resolution, sensitivity, and calibration than SETHI. This made it an ideal dataset for further shell identification activities.

\section{Procedure}

This summer, our project consisted of visually identifying new HI Shells using data downloaded from the GALFA-HI online database. For each data cube, we visually searched for new shells, compared my discovered shells with catalogs of known shells, and generated a new catalog of previously unknown shells including measurements of their basic properties.

We began by creating custom cubes in which to search for shells. The data used in this project are publicly available online via the GALFA-HI website (https://sites.google.com/site/galfahi/Home). This survey covers the region of the sky visible from the Arecibo Telescope. HI maps are available for regions with celestial coordinates $0^{\mathrm{h}}$ to $24^{\mathrm{h}}$ in Right Ascension and $-2^{\circ}$ to $+38^{\circ}$ in Declination, although data are complete for only about $1 / 3$ of the sky, and is not contiguous in all regions. Note that Right Ascension \& Declination are the sky equivalents of longitude and latitude here on Earth.

Each custom data cube covered a portion of the Arecibo sky approximately $20^{\circ} \times 20^{\circ}$ in size. At every location in the sky, the amount of $21-\mathrm{cm}$ emission has been measured for all radial velocities corresponding to motions of Galactic gas. Because of its orbits around the Galactic center, gas at different distances from us will be detected at different radial velocities. In addition, shell expansion, as well as turbulent and streaming motions affects the observed velocities. The data cubes that were created contain $512 \mathrm{HI}$ maps at radial velocities spaced by $0.74 \mathrm{~km} / \mathrm{s}$. These cubes overlapped in position in order to detect shells which could potentially lie on the edge of the cube.

Each cube was then methodically searched for potentially undiscovered shells. Visual identification was the preferred method due to other search methods excluding shells that are oddly shaped or extreme in size, and excluding older non-expanding shells. A majority of the work being done for this project utilized the $<$ kvis $>$ software of the Karma Toolkit (Gooch 1996) in order to satisfy the project goals. This general purpose image and movie viewer is capable of loading multiple data sets and displaying multiple windows. These features allow one to step through the various velocity slices and to adjust histogram and pseudo color settings in order to highlight features in both faint $\&$ bright gas regions. Each cube was searched using multiple display settings in order to conduct a complete analysis of the data.

Although the online GALFA database is currently incomplete, the existing data proved to be more than sufficient for a significant number of new potential shells to be identified and measured.

Upon discovering a potential shell, we recorded its center coordinates, the reference velocity at which it is most shell-like, its right ascension, and its declination. After recording the findings for a particular cube, we compared the shell candidates with catalogs of previously known shells. This allowed for the determination of which of the shells were previously unknown. 
Each of the newly discovered shells was further analyzed using $<$ kvis $>$. This included measuring quantitative values such as the minimum and maximum values for Right Ascension, Declination and velocities at which it appeared shell-like, We also recorded qualitative data regarding the behavior of the potential shell while stepping through the velocity slices. These data were used to create a GSH ID (an identifier for a specific shell using the Galactic coordinate system) and to tabulate a mean angular diameter and shape parameter for each shell. The mean angular diameter $\left(\Delta \theta_{\text {avg }}\right)$ is a basic size description based on the lengths of the short and long axes, assuming the shell is elliptical in shape. By determining four points within the shell walls at the end of each axis, we computed the axis lengths, $\Delta \theta_{1}, \Delta \theta_{2}$ (the distance between each set of points), and the mean angular diameter (the average of the two distances). Using the same four boundary points, we also calculated the shape parameter, a value that specifies the eccentricity of a given shell. For our purposes, we've defined the shape parameter to be a value ranging from 0 to 1 where 0 represents a straight line and 1 represents a perfect circle $\left[S=1-\left(\Delta \theta_{1}-\Delta \theta_{2}\right) / 2 \Delta \theta_{\text {avg }}\right]$. We also determined the range of velocities at which the feature is shell-like, and made an image of the shell at its reference velocity. An example of such an image can be seen in Figure 1. This process was repeated over and over in order to create a large catalog of previously unknown potential shells.

\section{GSH 167-13-042}

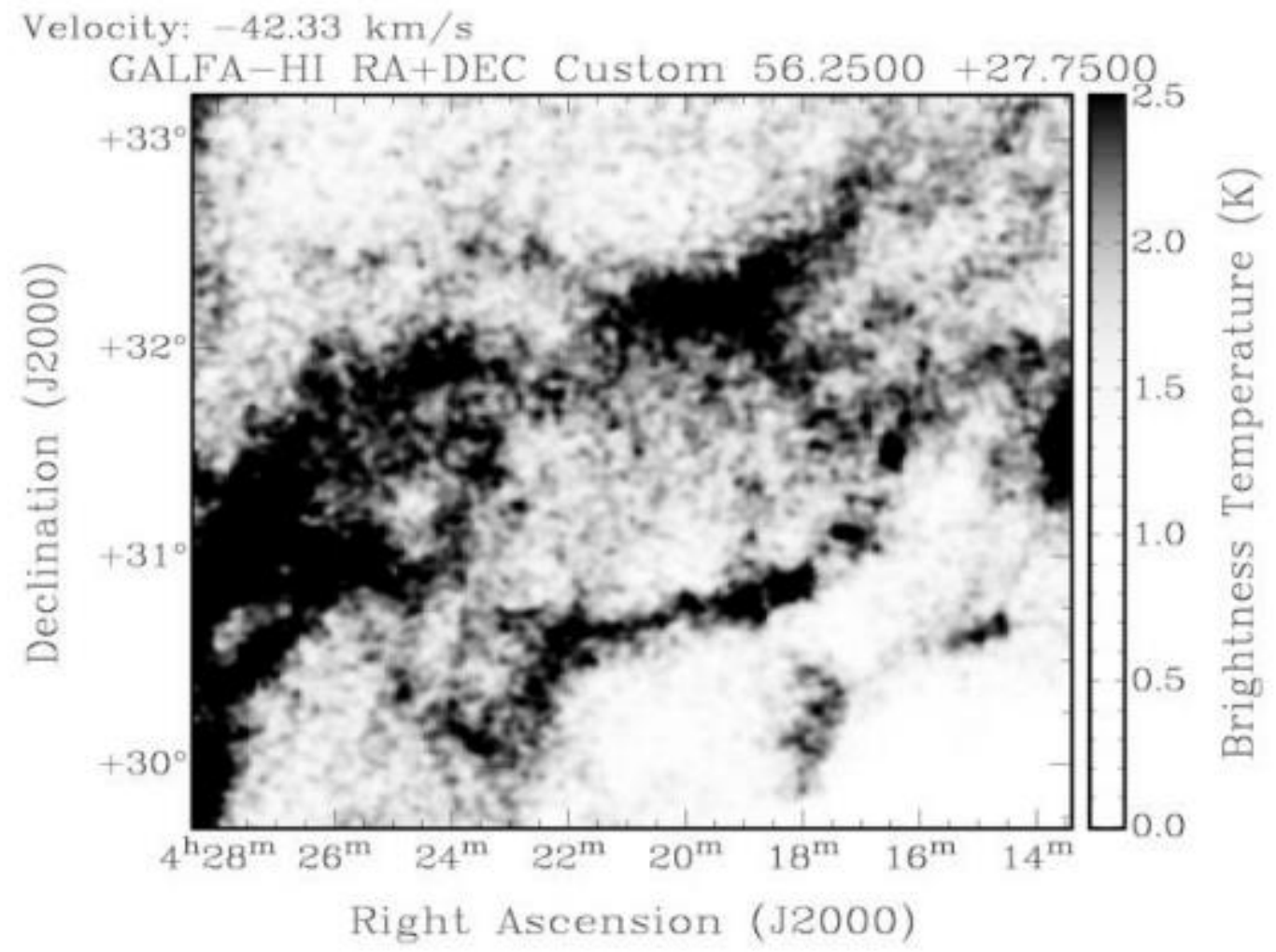

Figure 1: Image produced in <kvis> of a Neutral Hydrogen (HI) Shell discovered in the $21 \mathrm{~cm}$ GALFA-HI database. We can see that it is centered at $04 \mathrm{~h} 21 \mathrm{~m}$ in Right Ascension and $31.3^{\circ}$ in Declination. It's reference velocity is $-42.33 \mathrm{~km} / \mathrm{s}$ and it has a Mean Angular Diameter of $1.79^{\circ}$. 


\section{Results}

The results of this project include a new list of previously undiscovered shell-like HI features along with measurements of their basic properties, and images of each feature. The search process utilized six data cubes and resulted in the identification of 141 potential shells. While the total number of shells discovered was 151, ten of these shells were not included in the final list. Of these ten, seven were already listed in online catalogs and were confirmed as previously identified, while the remaining three features were considered to be insufficiently shell-like for inclusion.

Table 1 displays sample data regarding 7 of the shells. The first column contains the GSH identifier based on the shell's Galactic coordinates. For each shell, the table includes celestial coordinates for the center of the shell (RA, Dec, columns 2-3), and the Mean Angular Diameter and Shape values (columns 4-5), which are a direct result of four measured boundary points, as described in the Procedure.

The Quality Parameter in the final column is a summary of overall shell quality based on five shell parameters, each represented with a value between 0 and 1 . These factors, shown in columns 5-9, include the shape parameter (column 5), the completeness of the shell walls at the reference velocity (column 6), the extent to which the shell's shape and location remain constant over its velocity range (column 7), whether or not its front \& rear expanding walls are visible (column 8), and the fraction of the velocity range at which the shell appears relatively complete, as opposed to merely shell-like (column 9). For columns $7 \& 8$, the parameter will have a value of 1 if the feature behaves exactly as expected for an expanding shell, and 0 if it does not behave at all like an expanding shell. The final Quality value weights all 5 parameters equally, so is represented by a number between 0 and 5 , where 5 is a perfect shell, exhibiting all the features of an interstellar shell. Note that a shell with little expansion will not have obvious front and rear walls, so will be limited in quality. The quality of the potential shells ranged from values of 2.53 to 4.55 with an average value at 3.46 .

Table 1 - Measured Parameters for Selected Shells

\begin{tabular}{|c|c|c|c|c|c|c|c|c|c|}
\hline Official ID & $\begin{array}{c}\text { Center RA } \\
\text { (hh mm) }\end{array}$ & $\begin{array}{c}\text { Center Dec } \\
\text { (Deg) }\end{array}$ & MAD (Deg) & $\begin{array}{c}\text { Shape } \\
\text { Parameter }\end{array}$ & $\begin{array}{c}\text { Fraction } \\
\text { Closed }\end{array}$ & $\begin{array}{c}\text { Same } \\
\text { Shape/Location } \\
\text { at Multiple Vels? }\end{array}$ & $\begin{array}{c}\text { Front/Rear } \\
\text { Caps } \\
\text { Visible? }\end{array}$ & $\begin{array}{c}\text { Fraction of } \\
\text { Range over } \\
\text { which } \\
\text { completeness is } \\
\text { at Maximum }\end{array}$ & $\begin{array}{c}\text { Summary } \\
\text { (Quality } \\
\text { Parameter) }\end{array}$ \\
\hline GSH 171-11+017 & 0442 & 29.8 & 7.8 & 0.86 & 0.9 & 1 & 0.5 & 0.45 & 3.71 \\
\hline GSH 147-28-068 & 0231 & 30.4 & 0.56 & 1 & 1 & 1 & 0 & 0.5 & 3.5 \\
\hline GSH 169-28-016 & 0340 & 19.6 & 0.96 & 0.97 & 1 & 1 & 1 & 0.58 & 4.55 \\
\hline GSH 156-17+001 & 0331 & 35.7 & 3.4 & 0.55 & 0.8 & 0.8 & 0 & 0.38 & 2.53 \\
\hline GSH 162-13-027 & 0406 & 35.2 & 1.17 & 0.57 & 1 & 1 & 0.5 & 0.38 & 3.45 \\
\hline GSH 165-21-004 & 0349 & 27 & 2.33 & 0.66 & 1 & 0.8 & 0 & 0.36 & 2.82 \\
\hline GSH 173-19+017 & 0420 & 22.9 & 1.6 & 0.59 & 1 & 1 & 0.5 & 0.33 & 3.42 \\
\hline
\end{tabular}

The shells discovered during this project vary greatly in size and shape. Included in this table are the largest shell (GSH171-11+017), the smallest shell (GSH147-28-068), the highest quality shell (GSH169-28-016), and the lowest quality shell (GSH156-17+001). The remaining shells in the table are typical examples of the majority of potential shells discovered.

While many shells exhibited complete walls and a uniform circular shape, many also displayed odd or elongated shapes as well as incomplete walls or varying wall thickness. The mean angular diameter for the shells discovered in this search ranged from 0.56 to 7.80 degrees with a 
mean value of $1.85^{\circ}$. The shape parameter ranged from values of 0.42 to 1.00 with 0.79 representing the mean value. The distributions of mean angular diameters and shape parameters are shown in Figure 2. The left figure highlights the fact that a majority of the potential shells were small in size. It is likely that these shells were missed in previous searches due to the limited resolution of those data. The right figure tells us that most shells were relatively circular in shape. This is what we expect due to the fact that shape parameters near zero look increasingly less shell-like and hence aren't included in the final results.
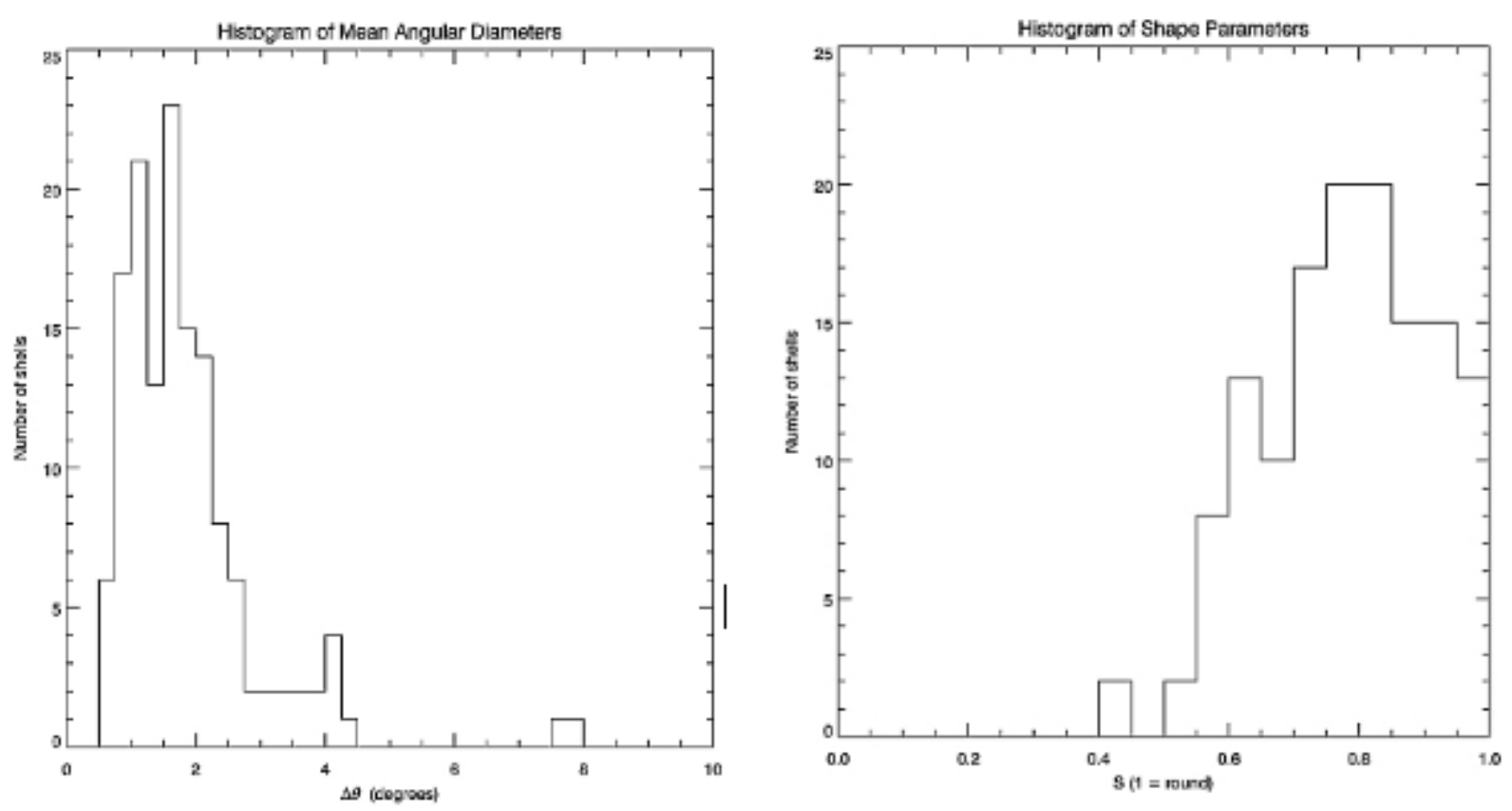

Figure 2: Histograms of Mean Angular Diameters (left) and Shape parameters (right) of neutral Hydrogen (HI) Shells discovered in the $21 \mathrm{~cm}$ GALFA-HI database. Mean angular diameters range from $0.56^{\circ}$ to $7.8^{\circ}$, with a majority of the potential shells $0.5^{\circ}<\theta<3^{\circ}$ in size. The average shape parameter was 0.79 . The lack of low shape parameters is due to line-like features not being considered sufficiently shell-like for inclusion in the catalog.

Many shells also displayed evidence for expansive behavior. When stepping through the velocity slices, a clearly expanding shell will contain both a front and rear wall at the maximum and minimum velocity values respectively. Shells which are considered to show signs of expansion will display at least one of these two walls. Shells without such features may be older shells whose expansion has slowed over time and are in the process of cooling and mixing with their surroundings. However, it is also possible that these features are absent in various shells because they contain low density walls that are only visible along the edges.

Of the total number of shells discovered (141), 100 displayed possible signs of expansion by appearing to exhibit at least one front or rear wall. If this were accurate (see below), we could conclude that roughly $71 \%$ of the shells observed were still in the expansion process while the remaining 29\% are older shells whose expansion has slowed or halted over time. However this is likely an overestimate. Upon viewing the GALFA data, it becomes apparent that the vast number of random interstellar features could have been mistaken for a shell wall in many cases. 
When we examine the 100 potential shells which showed possible signs of expansion, only three shells contained both a front and rear wall. It is very likely however, that these are indeed expanding shells. It is evident that shells which are obviously expanding do not represent the majority of features observed. Roughly $2 \%$ of the total 141 shells were found to contain both a front and rear wall. This supports the idea that visual identification is not biased towards expanding features.

It is clear that the observed "single-wall" potential shells will require re-examination in order to accurately determine if they qualify as shells displaying signs of expansion or were simply mistaken as such. Such future work will entail the study of changes in shell size on either side of the reference velocity, and examination of the data in position-velocity space to search for further evidence of expansion.

We created a sky map displaying locations and relative sizes of the newly identified potential shells. Figure 3 plots the shells using the equatorial coordinate system and ranges from 0 to 6 hours in Right Ascension and 16 to 40 degrees in Declination. Although the shells are represented as perfect circles on the figure, we must note that they are in fact irregular. The relative sizes are based on the mean angular diameter of the respective shells. Upon viewing the figure, we can clearly see a higher number of potential shells being identified proximate to the Galactic plane. Near the left side of the diagram, we see a vertical dashed line located at 5.25 hours in Right Ascension. This line represents the edge of the data searched, hence there were no potential new shells discovered beyond this boundary (Right Ascension > 5.25 hours).

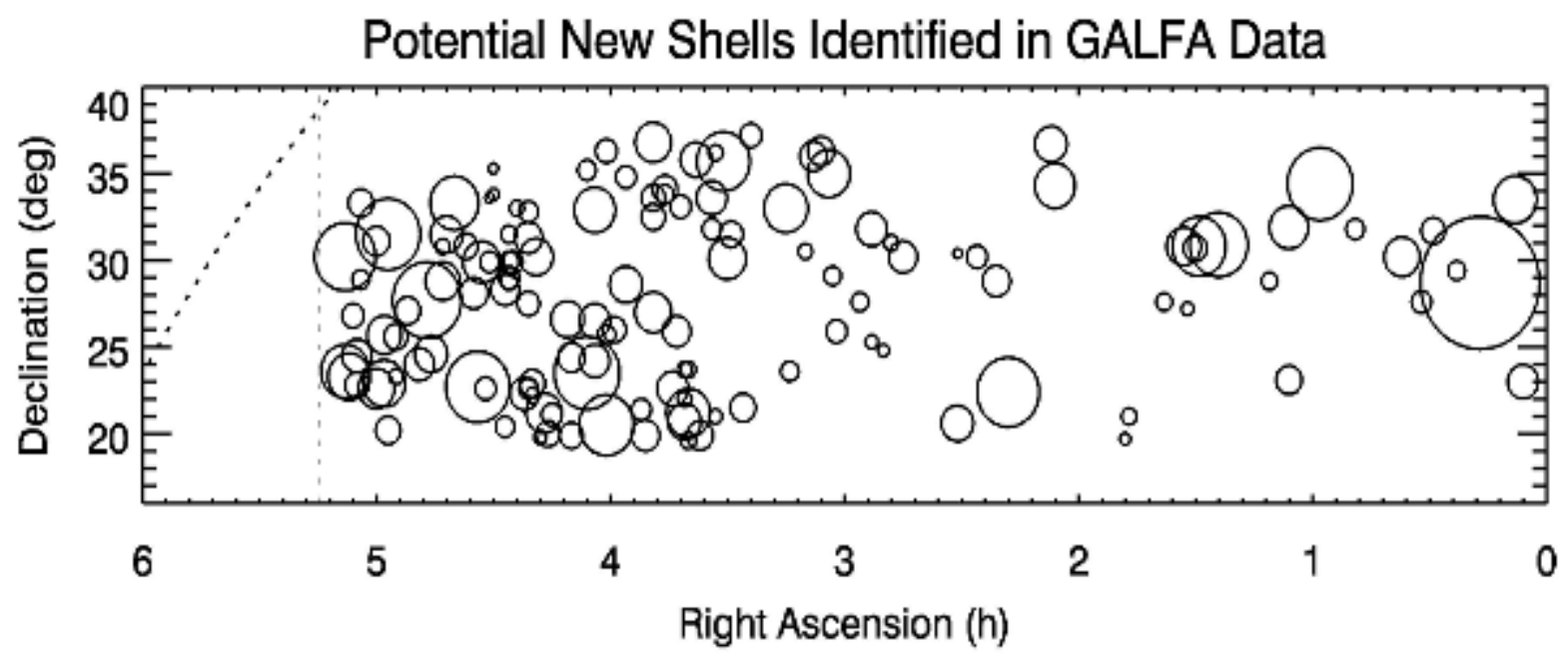

Figure 3: Sky map of neutral Hydrogen (HI) Shells discovered in the $21 \mathrm{~cm}$ GALFA-HI database. We can see that it ranges from $0 \mathrm{~h}$ to $6 \mathrm{~h}$ in Right Ascension and $16^{\circ}$ to $40^{\circ}$ in Declination. Each circle charts the location and relative size of the respective potential shell. The diagonal dashed line on the far left of the figure represents the location of the $\mathrm{Galactic}$ plane while the vertical dashed line at 5.25 hours Right Ascension represents the "edge" of the data searched. 
In addition, a common object resembling a "figure-eight" design was observed multiple times in various data cubes. A total of seven of these objects were discovered (seven figure-eights, fourteen potential shells). Each figure eight showed a consistent trend of being composed of small, complete, circular shells which were clearly related to one another upon stepping through their velocity range. And example of such an object can be observed in Figure 3.

\section{GSH 141-41-064 / GSH 140-40-064}

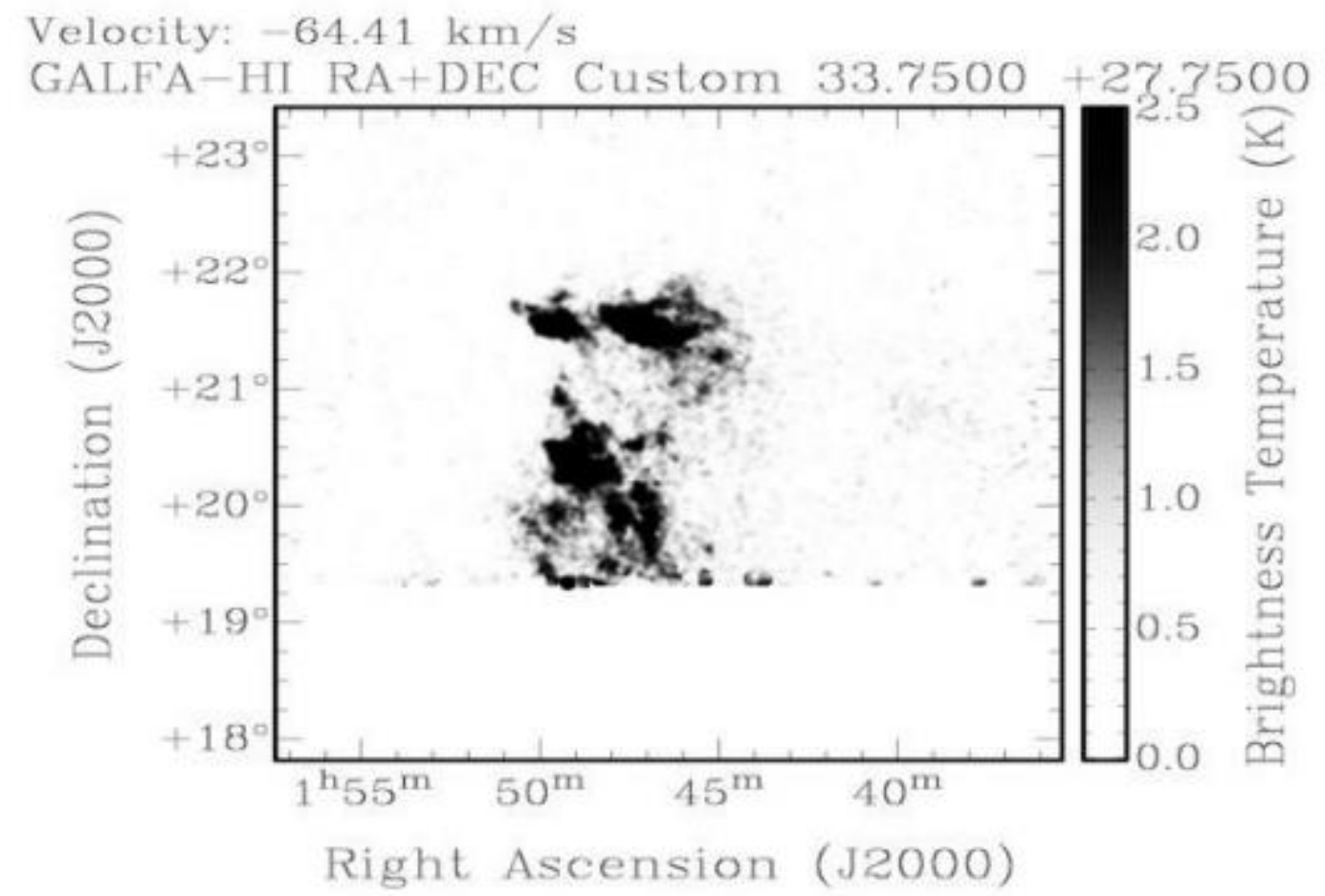

Figure 4: Image produced in Kkvis> of a "Figure-eight" pair of shells discovered in the $21 \mathrm{~cm}$ GALFA-HI database. We can see that the object is composed of two distinct shells; one centered at $01 \mathrm{~h} 48 \mathrm{~m}$ in Right Ascension and 19.6 degrees in Declination, while the other is centered at $1 \mathrm{~h} 47 \mathrm{~m}$ in Right Ascension and 21 degrees in Declination.

\section{Conclusions}

This project has expanded the current database of potential HI Shells in our Galaxy. A more complete list of HI shells will provide important data in regards to the study of shell evolution and the role of shells within the ISM. The visual identification methods that were used allowed for the identification of many shells which undoubtedly would have been missed by a computer search.

The mean angular diameter data for the newly discovered shells reveals that a majority of the shells are small in angular size. Many large shells have been previously discovered by visual searches, making the identification of small shells a priority with regards to completing the 
overall picture of shell evolution. While a primary advantage of visual searches is the ability to identify odd-shaped shells, such features must also be "sufficiently shell-like" in order to qualify as a shell at all. The shape parameter data reveals that a typical potential shell included in our catalog is slightly elliptical, however some shells were measured to be extremely analogous to a perfect circle. By definition, features with a low shape parameter would appear line-like and therefore would not qualify as a potential shell for our catalog.

The high number of small shells discovered can be attributed to the improved angular resolution of the GALFA-HI survey as compared to previous surveys. Many of the shells were in fact too small to resolve prior to the release and examination of these data. We identified a very large number of new features per data cube, which then limited the overall number of cubes that could be searched within the time frame. The study of these small, previously unknown shells will be key to completing the overall picture of shell evolution.

The completion of this project also gives one a good perspective regarding the time required to complete the search of a given data cube. Even as the search procedure was optimized with repetition, the search process in general required more time than originally anticipated and as a result, fewer cube searches were completed. Approximately $1 / 7^{\text {th }}$ of the total amount of data cubes containing GALFA data were searched, revealing the vast amount of potential future work yet to be completed. Additionally, as the project progressed, the cubes being searched were increasingly closer to the Galactic plane, yielding more shells per cube as opposed to those cubes farther from the Galactic plane. The sky map (See figure 3) that was generated displays this result as well as the relative sizes of the shells.

It is also likely that a large number of small potential shells were missed by the initial search, solely due to the overwhelming number of features now visible with the improved angular resolution. Later study of these data will not only include the search of the remaining data cubes, but also the reinvestigation of each cube by another set of eyes. This will likely add features to our catalog which were initially missed, resulting in a more complete search by eliminating bias. It will be interesting to compare the results of two visual searches of the same data by different observers.

It will also be crucial to calculate the kinematic distances to the potential shells. Because the data provides an angular view of the sky, it is impossible to determine whether a shell that is measured to be small is actually small in size or simply appears small due to its distance from Earth. Following the computation of kinematic distances, it will be possible to estimate the actual shell sizes, the energy required for the shells to form, and the approximate age of any expanding shells.

We also need to re-examine the signs of expansion properties for each potential shell. The initial search revealed an unusually high number of potential shells with at least some possible signs of front or rear walls. Expanding shells should appear to have different sizes at different velocities, and their expansion should also be visible in position-velocity space. By determining whether these features are actually shell walls or just random interstellar features, we can more accurately classify these potential shells as expanding or not expanding. Complete data on shell sizes, ages, energy required to form, and expansion properties is necessary to understanding their role in Galactic evolution. 


\section{Acknowledgments}

I would like to acknowledge the WSGC for granting me this award and allowing me this opportunity to further my education and involvement in Astronomy research.

We acknowledge the use of the GALFA-HI database (https://sites.google.com/site/galfahi/), which uses data acquired at Arecibo Observatory. The Arecibo Observatory is operated by SRI International under a cooperative agreement with the National Science Foundation (AST1100968), and in alliance with Ana G. Méndez-Universidad Metropolitana, and the Universities Space Research Association.

This research has also made use of the $<$ kvis $>$ software of the Karma Toolkit.

In addition, this research has made use of the SIMBAD database and the VizieR catalogue access tool operated at CDS, Strasbourg, France.

\section{References}

Cox, D. P. 2005, ARA\&A, 43, 337.

Ehlerová, S. \& Palouš, 2005, Astron. \& Astrophys., 437, 101.

Gooch, R. 1996, Astronomical Data Analysis Software and Systems V, 101, 80.

Hartmann, D., \& Burton, W. B. 1997, Atlas of Galactic Neutral Hydrogen (Cambridge University Press), ISBN 0521471117.

Korpela, E. J., Demorest, P., Heien, E., Heiles, C., \& Werthimer, D. 2002, Seeing Through the Dust: The Detection of HI and the Exploration of the ISM in Galaxies, 276, 100.

Korpela, E. J., Demorest, P., Heien, E., Heiles, C., \& Werthimer, D. 2004, How Does the Galaxy Work?, 315, 97.

McKee, C. F., \& Ostriker, J. P. 1977, ApJ, 218, 148.

Peek, J. E. G., Heiles, C., Douglas, K. A., et al. 2011, ApJS, 194, 20.

Shapiro, P. R., \& Field, G. B. 1976, ApJ, 205, 762.

Slavin, J. D., \& Cox, D. P. 1993, ApJ, 417, 187. 